\title{
Structural Changes in the Duration of Bull Markets and Business CyCle DYNAMICS*
}

\author{
João $\mathrm{Cruz}^{a}$, João Nicolau ${ }^{b}$ and Paulo M.M. Rodrigues ${ }^{c}$ \\ ${ }^{a}$ ISEG-Universidade de Lisboa \\ $b$ ISEG-Universidade de Lisboa and CEMAPRE \\ ${ }^{c}$ Banco de Portugal and Nova School of Business and Economics, Universidade Nova de Lisboa
}

November, 2020

\begin{abstract}
This paper tests for structural changes in the duration of bull regimes in 18 developed and emerging economies' adjusted market capitalization stock indexes, by using the novel approach of Nicolau (2016) as well as two additional new procedures introduced here; and investigates whether the structural changes detected in the bull markets' duration are connected to the business cycle. We conclude that changes in the duration of bull market regimes seem to precede periods of economic recession. The results provide statistically significant evidence that decreases in bull markets' duration do not occur independently from economic crises, as 13 out of the 18 markets considered in our sample verify such decreases at least 12 months prior to the occurrence of an economic crisis. Additionally, these structural changes seem to affect smaller companies first, and then the larger ones. The association between decreases in the bull market regimes' duration and economic crises is possibly a consequence of financial markets' leading behavior over the economy. These structural changes may serve as proxies for decreasing confidence in financial markets, which naturally affects economic stability.
\end{abstract}

Keywords: Structural breaks, duration, bull markets, business cycles.

JEL: C12, C22.

${ }^{*}$ João Nicolau was partially supported by the Project CEMAPRE - UID/MULTI/00491/2013 and PTDC/EGE-ECO/28924/2017 financed by FCT/MCTES and Paulo M. M. Rodrigues through project PTDC/EGE-ECO/28924/2017 and (UID/ECO/00124/2013 and Social Sciences DataLab, Project 22209), POR Lisboa (LISBOA-01-0145-FEDER-007722 and Social Sciences DataLab, Project 22209) and POR Norte (Social Sciences DataLab, Project 22209 


\section{Introduction}

Bull and bear market regimes have been characterized as long periods of price rises and price declines, respectively (Chauvet and Potter, 2000 and Sperandeo, 1990). The identification of these regimes is important for policy makers and for investors, given that their impact on asset pricing is an important source of time variation in risk premia (see, for example, Gordon and St-Amour, 2000; Ang et al., 2006). Over the years, this has led to the development of parametric and non-parametric methodologies for the identification of bull and bear markets (see e.g. Kole and van Dijk, 2017). A first-class of approaches proposed in the literature considered data-based identification methodologies, which are mainly concerned with converting the notion of rising and declining stock prices into quantitative criteria to enable the construction of identification algorithms; see, for instance, Fabozzi and Francis (1977) and Kim and Zumwalt (1979). However, these approaches rely on returns sharing some common underlying characteristics throughout the entire sample (such as, e.g., a common mean or standard deviation), and identify bull and bear markets as extremes within this set of returns. A second-class of approaches, which is less restrictive, considers the identification of bull and bear regimes as periods during which prices are not too far from local peaks and troughs of the current market. Hence, bull and bear markets are detected relative to characteristics of the current market and not the entire sample. This approach has been used by, among others, Pagan and Sossounov (2003), Lunde and Timmermann (2004) and Candelon, Piplack and Straetmans (2008). Kole and van Dijk (2017) provide an extensive comparison of different approaches and an in-depth discussion of their merits.

Regime duration dependence has also been an active topic of research, both in the business cycle (Chauvet and Potter, 2009) as well as in the financial markets (Pagan and Sossounov, 2000) literature. For the purpose of the present paper we will focus on duration dependence in bull markets only and employ the algorithm proposed by Lunde and Timmermann [LT] (2004) for the identification of these markets. Our first contribution lies in the detection of possible structural changes in duration dependence. Specifically, we are going to test whether bull market duration is constant over time. This is an important point to which to date little attention has been given in the literature. Its importance is directly linked to applications of bull and bear regimes as key components of stock markets. In particular, if a structural change in the cycle duration is wrongly left unconsidered, then an analysis based on bull and bear markets will most likely be compromised. Moreover, we use the estimated dates associated to the detected structural changes to further link these with business cycle peaks and troughs.

The literature on structural change tests in duration is very scant. One of the few approaches available to test for changes in duration dependence is the procedure introduced by Nicolau (2016) who provides an analysis of structural changes in duration dependence in the Dow Jones Industrial Average index. In this paper we will use and extend the 
approach of Nicolau (2016). In the next section we will briefly introduce this procedure, along with two alternative tests derived from it.

A further important point which has received considerable attention in the literature is the link between macroeconomics and finance, especially after the financial crisis of 2008 which had worldwide impact. Building on the works of, e.g., Estrella and Mishkin (1998), Avouyi-Dovi and Matheron (2005), Claessens et al. (2012) and Nyberg (2013), a second contribution of this paper is to explore the possible relationship between structural changes in the duration of bull markets and business cycles. To the best of our knowledge, the literature is silent on the impact of structural breaks in the duration of bull and bear cycles. Still, research on duration dependence is available, with early examples provided by Cochran and Defin (1995), who use parametric hazard models to investigate duration dependence in US stock market cycles from January 1885 to July 1992. Their results show that duration dependence exists in pre-World War II expansions and in post-World War II contractions. Sichel (1991) uses a parametric hazard model and shows that expansions became longer, on average, after World War II, while contractions became shorter.

Understanding stock market regimes and economic cycles and how they are connected to investment performance can help determine the best timing strategies and portfolio composition. Empirical evidence suggests that, typically stocks fall prior to recessions. This synchronization of stock market fluctuations and business cycles has received some attention by researchers. For example, Chauvet (1999) computes bull and bear market probabilities to predict cyclical turning points in economic activity. Moreover, using Asian data, Candelon and Metiu (2011) show that financial cycles lead business cycles by six months on average.

However, while stocks as a whole have leading behavior relative to the economy, specific sectors and firms may have different relative performance throughout the economic cycle. Depending on the business activities of a given sector or industry, there is generally a particular phase of the business cycle that is more favorable to some activities/firms/sectors than others (see e.g. Fort et al., 2013). The third contribution of this paper looks to shed light on this topic by considering small, mid and large cap stocks in our analysis. This is of importance since periods of market upheaval and economic recession are characterized by investor flight to perceived quality and liquidity in response to uncertainty and fear. Many investors reduce their overall exposure to equities during times of crisis. Others reduce or sell off their exposure to the small cap segment of the market. Market cap is one measure of potential liquidity for stocks, and some investors sell off their small cap holdings during these volatile periods, reinvesting the proceeds in what they believe to be safer and more liquid assets. Moreover, smaller companies may be better positioned to move quickly as the economic environment improves, which suggests that these companies' bull market cycles may have leading behavior when compared to medium and large companies' bull market regimes. 
The underlying motivation for this work relies on a central premise of finance theory, namely that financial markets are "forward looking." Since news and information about future states of the economy are continuously processed by market participants, expectations about upcoming economic conditions as well as risk preferences and tolerances are also subject to continuous revision. Such revisions may give rise to inducements to trade, which causes relative stock prices and stock market indexes to fluctuate. Given that trading levels are directly related to liquidity, one may expect that aggregate liquidity should also convey information about future macroeconomic conditions. For example, the "flight to quality" phenomenon, which reflects the "forward looking" nature of equity markets, usually occurs prior to difficult economic times when investors shift their equity allocation to move away from the stock market or invest into safer securities to construct portfolios that are more defensive and more focused on wealth preservation. During a "flight to quality" episode, an unusual amount of asset trading occurs in a short period of time which leads to important price changes and enhanced stock volatility, which in turn causes aggregate liquidity to worsen (illiquidity increases). In a recent study, Næs et al. (2011) suggest that stock market liquidity acts as a strong leading indicator of economic growth.

A bull market coming to an end does not necessarily mean an upcoming economic recession. However, our most novel finding is that periods of structural changes associated with decreases in duration of bull market regimes seem to precede periods of economic recession. Hence, the present study aims to contribute to the understanding of the link between finance and macroeconomics, by exploring the possible relations between structural changes in the duration of bull markets and the business cycle, a research topic not approached to date. The paper is organized as follows. Section 2 introduces the duration dependence measure and structural change tests considered; Section 3 provides the results of an in-depth Monte Carlo analysis of the empirical performance of the tests, i.e. provides information on the finite sample properties (empirical size and power) of the procedures proposed in this paper. Section 4 presents the structural breaks tests' results obtained from the adjusted market capitalization stock indexes and analyses the link between duration dependence in bull cycles and economic recessions; and Section 5 concludes.

\section{Breaks in duration dependence}

\subsection{Bull markets duration}

A crucial step for the detection of possible structural changes in the duration of bull markets consists in the identification of the bull regimes. There are several (parametric and nonparametric) approaches in the literature which allow for the identification of 
bull and bear markets. Kole and van Dijk (2017) show that non-parametric rule-based methods are generally preferable for (in-sample) identification of the state of the market, as they are more transparent and robust to misspecification than alternative methods. Thus, in this paper, the algorithm proposed by LT is preferred, given that it does not restrict cycle duration, and avoids interval censoring issues. This algorithm defines bullish cycles as the movements of a time series between two local maximums without significant drops in between, or as the movements between a local minimum and a local maximum.

The algorithm considers a change from a bull (bear) to a bear (bull) regime if the price drops (increases) by more than a pre-specified percentage. Specifically, the approach works as follows: Let the stock market be in a bullish state at time $t=t_{0}$, with $P_{t_{0}}^{\text {Max }}$ equal to its value at that period $\left(P_{t_{0}}\right)$ and consider the stopping time variables $\tau_{\text {Max }}$ and $\tau_{\text {Min }}$ such that,

$$
\begin{aligned}
& \tau_{\text {Max }}\left(P_{t_{0}}^{\text {Max }}, t_{0} \mid I_{t_{0}}=1\right)=\text { inf }\left\{t_{0}+\tau: P_{t_{0}+\tau} \geq P_{t_{0}}^{\text {Max }}\right\} \\
& \tau_{\text {Min }}\left(P_{t_{0}}^{\text {Max }}, t_{0} \mid I_{t_{0}}=1\right)=\text { inf }\left\{t_{0}+\tau: P_{t_{0}+\tau}<\left(1-\lambda_{2}\right) P_{t_{0}}^{\text {Max }}\right\} .
\end{aligned}
$$

If $\tau_{\text {Min }}<\tau_{\text {Max }}$, then a change of state is recognized in period $t_{0}+1$ such that periods $t_{0}+1, \ldots, t_{0}+\tau_{\text {Min }}$ correspond to bearish states. $\left(1-\lambda_{2}\right)$ is a pre-specified percentage where $\lambda_{2}$ is a user-defined parameter (in the empirical analysis below we set $\lambda_{2}=0.15$ ). Change from bear to bull states are analogously defined.

There are two main implementation issues related to the LT-algorithm: first, the choice of filters, and second, the short-term fluctuations and filtering. If there is a drift in the stock price series from which one derives the bull/bear markets, one has to adjust the filter so as to account for this feature. In particular, if the series exhibits an upward trend, an asymmetric filter is required so that in order to go from a bear market to a bull market, the stock price would have to increase more than it would have to decrease to go the other way (see LT for details).

Once the bull markets are identified their duration dependence can be computed. For that purpose, consider the indicator variable $S_{t}$, which takes a value of one if the stock market is in a bull state at time $t$, and zero otherwise (bear state). Assuming, as in Nicolau (2016), that $\left\{S_{t}\right\}$ is a stationary first order Markov chain process, the duration of the bull market is determined as,

$$
\theta:=\left(1-p_{11}\right)^{-1}
$$

where $p_{11}$ is the transition probability, i.e., $p_{11}:=P\left(S_{t}=1 \mid S_{t-1}=1\right)$; see, e.g., Taylor and Karlin (1998). The duration of the cycle is estimated by replacing $p_{11}$ with its maximum likelihood estimate, viz.,

$$
\widehat{p}_{11}:=\frac{n_{11}}{n_{1}}
$$

where $n_{1}$ is the number of times $S_{t}=1$ in a given sequence and $n_{11}$ is the number of times that $S_{t}=1$ given that $S_{t-1}=1$ (see, for example, Basawa and Rao, 1980). 


\section{$2.2 \quad$ The structural change tests}

To determine whether bull market durations are constant over time, a recent procedure introduced by Nicolau (2016) is considered and extended. In specific, considering $\theta_{t}$ the duration of a bull cycle at time $t$ as defined in (2.3) and focusing on observations $t:=\lfloor r T\rfloor$ for $r \in\left[r_{0}, r_{1}\right]$, a pre-specified compact subset of $(0,1)$, where $\lfloor x\rfloor$ is the integer part of $x$ and $T$ is the sample size, our target is to test $H_{0}: \theta_{\lfloor r T\rfloor}=\theta, \forall r \in\left[r_{0}, r_{1}\right]$ (i.e. parameter constancy) against the alternative $H_{1}: \theta_{\lfloor r T\rfloor} \neq \theta$ for some $r \in\left[r_{0}, r_{1}\right]$.

The structural breaks tests considered are:

$$
\begin{aligned}
& \mathcal{D}_{1}:=\underset{r \in\left[r_{0}, r_{1}\right]}{\operatorname{Max}} Q_{T}^{2}(\lfloor r T\rfloor) ; \\
& \mathcal{D}_{2}:=\frac{1}{T} \sum_{j=w+1}^{T-1} Q_{T}^{2}(j) ; \\
& \mathcal{D}_{3}:=\frac{1}{T} \sum_{j=w+1}^{T-1}\left|Q_{T}(j)\right|
\end{aligned}
$$

where

$$
Q_{T}(\lfloor r T\rfloor):=\left(\frac{\lfloor r T\rfloor-w}{T-w} \frac{\lfloor r T\rfloor}{\widehat{\sigma}^{2}}\right)^{1 / 2}\left(\widehat{\theta}_{\lfloor r T\rfloor}-\widehat{\theta}_{T}\right) .
$$

The constant $w$ in (2.8) is a shifting value such that $w<\left\lfloor r_{0} T\right\rfloor$ and $\widehat{\sigma}^{2}$ is the maximum likelihood estimate of,

$$
A \operatorname{Var}\left(\widehat{\theta}_{T}\right):=\lim _{T \rightarrow \infty} \operatorname{Var}\left(\sqrt{T}\left(\widehat{\theta}_{T}-\theta\right)\right)=\frac{p_{11}}{\left(1-p_{11}\right)^{3} \pi_{1}}
$$

with $\pi_{1}:=P\left(S_{t}=1\right)$. The test in (2.5) was introduced in this context by Nicolau (2016) and is based on Andrews (1993), and the tests in (2.6) and (2.7) introduced in this paper are inspired in Andrews and Ploberger (1994).

Moreover, under the regularity conditions layed out in Nicolau (2016) it follows from the continuous mapping theorem, as $T \rightarrow \infty$, that,

$$
\begin{aligned}
& \mathcal{D}_{1} \stackrel{d}{\rightarrow} \underset{r \in R}{\operatorname{Sup} B(r)^{2} ;} \\
& \mathcal{D}_{2} \stackrel{d}{\rightarrow} \int_{0}^{1} B(r)^{2} d r ; \\
& \mathcal{D}_{3} \stackrel{d}{\rightarrow} \int_{0}^{1}|B(r)| d r
\end{aligned}
$$

where $B(r):=W(r)-r W(1)$, and $W(r)$ is a standard Wiener process. For detailed proofs of these results see, e.g., Nicolau (2016).

Critical values at the $10 \%, 5 \%$ and $1 \%$ significance levels for the tests in (2.5), (2.6) and (2.7), are $1.46,1.78,2.54$, for $\mathcal{D}_{1}, 0.34,0.45,0.75$, for $\mathcal{D}_{2}$, and $0.49,0.58,0.76$ for $\mathcal{D}_{3}$, respectively. 


\section{Monte Carlo Simulation Study}

In this section we evaluate the finite sample performance of the test statistics in (2.3) (2.5) through Monte Carlo analysis to study the statistical properties of the novel tests proposed in Section 2.2. Throughout the simulation study the number of replications used is $N=10000$ and the performance of the tests is evaluated at a $5 \%$ nominal significance level. For the development of the simulation study it is important to note that the performance of the tests depends on several factors, including the data generation process (DGP), the sample size and whether $\hat{\sigma}^{2}=\widehat{A \operatorname{Var}}\left(\widehat{\theta}_{T}\right)$ or $\tilde{\sigma}^{2}=\widehat{A V a r}\left(\tilde{\theta}_{\lfloor r T\rfloor}\right)$ is used to compute the test statistics.

To simulate the empirical size of the tests the DGP considered is,

$$
\left\{\begin{array}{l}
p_{11}:=P\left(S_{t}=1 \mid S_{t-1}=1\right)=\alpha ; t=1, \ldots, T \\
p_{00}:=P\left(S_{t}=0 \mid S_{t-1}=0\right)=\beta ; t=1, \ldots, T
\end{array}\right.
$$

where $(\alpha ; \beta)=\{(0.99 ; 0.99),(0.95 ; 0.95)\}, T=\{3000,6000,15000\}$, and where either $\hat{\sigma}^{2}$ or $\tilde{\sigma}^{2}$ is used.

For the analysis of the finite sample power of the three tests we used the DGP,

$$
\left\{\begin{array}{l}
p_{11}^{1}:=P\left(S_{t}=1 \mid S_{t-1}=1\right)=\gamma ; \\
p_{00}^{1}:=P\left(S_{t}=0 \mid S_{t-1}=0\right)=\delta ;
\end{array} \quad \text { for } t=1, \ldots, T_{b r e a k}-1\right.
$$

and

$$
\left\{\begin{array}{c}
p_{11}^{2}:=P\left(S_{t}=1 \mid S_{t-1}=1\right)=\lambda ; \\
p_{00}^{2}:=P\left(S_{t}=0 \mid S_{t-1}=0\right)=\psi ; \quad \text { for } t=T_{\text {break }}, \ldots, T
\end{array}\right.
$$

where $(\gamma ; \delta ; \lambda ; \psi)=\{(0.996 ; 0.99 ; 0.99 ; 0.99),(0.99 ; 0.99 ; 0.996 ; 0.99),(0.98 ; 0.95 ; 0.95$; $0.95),(0.95 ; 0.95 ; 0.98 ; 0.95)\}$. Also in this case $T=\{3000,6000,15000\}$ and $\widehat{\sigma}^{2}$ as well as $\tilde{\sigma}^{2}$ are used. Additionally, $T_{\text {break }}:=\lfloor\tau T\rfloor$ with $\tau=(0.5,0.8)$.

To develop the Monte Carlo simulations we implement the following steps:

(1) Generate a sample of size $\mathrm{T}$ for a continuous variable $\mathrm{U} \sim \mathrm{Uniform}(0,1)$;

(2) Initialize the process $\left\{S_{t}\right\}$ with regard to the initial probabilities specified taking into account that:

$$
\left\{\begin{array}{l}
p_{1}^{(1)}:=P\left(S_{1}=1\right)=\frac{p_{01}}{1-\left(p_{11}-p_{01}\right)} \\
p_{0}^{(1)}:=P\left(S_{1}=0\right)=\frac{p_{10}}{1-\left(p_{00}-p_{10}\right)}
\end{array}\right.
$$

and

$$
\begin{cases}S_{1}=1 \quad \text { if } \quad U_{1} \leq p_{1}^{(1)} \\ S_{1}=0 \quad \text { if } \quad U_{1}>p_{1}^{(1)}\end{cases}
$$

(3) Considering the transition probabilities in step (2), generate $\left\{S_{2}, S_{3}, \ldots, S_{T}\right\}$ as,

$$
\left\{\begin{array}{l}
S_{t}=1 \quad \text { if } \quad\left(S_{t-1}=1 \wedge U_{t} \leq p_{11}\right) \vee\left(S_{t-1}=0 \wedge U_{t}>p_{00}\right) \\
S_{t}=0 \quad \text { if } \quad\left(S_{t-1}=1 \wedge U_{t}>p_{11}\right) \vee\left(S_{t-1}=0 \wedge U_{t} \leq p_{00}\right)
\end{array}, \text { for } t=2,3, \ldots, T ;\right.
$$


(4) Based on $\left\{S_{1}, S_{2}, S_{3}, \ldots, S_{T}\right\}$ from step (3) compute $n_{1}$ and $n_{11}, t=w, w+1, \ldots, T$, and $\tilde{\sigma}^{2}$ and $\tilde{\theta}_{\lfloor r T\rfloor}$. Then, proceed to assemble $\left\{Q_{1, T}(w), Q_{1, T}(w+1), \ldots, Q_{1, T}(T)\right\}$.

(5) Calculate the test statistics for $\mathcal{D}_{1}, \mathcal{D}_{2}$ and $\mathcal{D}_{3}$;

(6) Repeat the previous steps $\mathrm{N}$ times.

(7) After obtaining the $\mathrm{N}$ test results associated with a given statistic, count the number of rejections of $H_{0}: \theta\lfloor r T\rfloor=\theta$ considering the specified nominal test size. The arithmetic mean of the number of rejections yields the empirical size/power of the test (depending on whether $H_{0}: \theta_{\lfloor r T\rfloor}=\theta$ holds or not).

Table 1: Empirical rejection frequencies under the null hypothesis

\begin{tabular}{ccccccc}
\hline & \multicolumn{3}{c}{$\widehat{\sigma}^{2}$} & \multicolumn{5}{c}{$\tilde{\sigma}^{2}$} \\
$\left(p_{11} ; p_{00}\right)$ & $\mathcal{D}_{1}$ & $\mathcal{D}_{2}$ & $\mathcal{D}_{3}$ & $\mathcal{D}_{1}$ & $\mathcal{D}_{2}$ & $\mathcal{D}_{3}$ \\
\hline & & \multicolumn{5}{c}{$\mathrm{T}=3000$} \\
$(0.99 ; 0.99)$ & 0.07 & 0.06 & 0.05 & 0.23 & 0.11 & 0.10 \\
$(0.95 ; 0.95)$ & 0.07 & 0.05 & 0.05 & 0.15 & 0.07 & 0.06 \\
& & & $\mathrm{~T}=6000$ & & \\
$(0.99 ; 0.99)$ & 0.07 & 0.05 & 0.05 & 0.20 & 0.09 & 0.08 \\
$(0.95 ; 0.95)$ & 0.07 & 0.05 & 0.05 & 0.11 & 0.06 & 0.06 \\
& & & $\mathrm{~T}=15000$ & & \\
$(0.99 ; 0.99)$ & 0.07 & 0.05 & 0.05 & 0.16 & 0.08 & 0.07 \\
$(0.95 ; 0.95)$ & 0.05 & 0.05 & 0.05 & 0.08 & 0.06 & 0.06 \\
\hline
\end{tabular}

The results in Table 1 suggest that the choice of $\widehat{\sigma}^{2}$ is relevant, noticing that $\tilde{\sigma}^{2}$ is associated with size distortions up to four times the nominal size for the test introduced by Nicolau (2016), and two times for the alternative tests. These distortions are mitigated if $\widehat{\sigma}^{2}$ is considered instead, with the empirical size being fairly close to the nominal one.

In addition, one may extrapolate that the number of state transitions influences the size properties of the given tests, with these exhibiting less over-rejection the more state transitions are verified in the sample (a similar conclusion can be drawn when analysing the statistical power; see results in Table 2). 
Table 2: Empirical rejection frequencies under the alternative

\begin{tabular}{|c|c|c|c|c|c|c|}
\hline \multirow[b]{2}{*}{$\left(p_{11}^{1} ; p_{00}^{1}\right) \&\left(p_{11}^{2} ; p_{00}^{2}\right)$} & \multicolumn{3}{|c|}{$\widehat{\sigma}^{2}$} & \multicolumn{3}{|c|}{$\tilde{\sigma}^{2}$} \\
\hline & $\mathcal{D}_{1}$ & $\mathcal{D}_{2}$ & $\mathcal{D}_{3}$ & $\mathcal{D}_{1}$ & $\mathcal{D}_{2}$ & $\mathcal{D}_{3}$ \\
\hline & \multicolumn{6}{|c|}{$\mathrm{T}=3000$ and $\tau=0.5$} \\
\hline$(0.996 ; 0.990) \&(0.990 ; 0.990)$ & 0.30 & 0.29 & 0.27 & 0.01 & 0.01 & 0.01 \\
\hline$(0.990 ; 0.990) \&(0.996 ; 0.990)$ & 0.01 & 0.07 & 0.09 & 0.47 & 0.44 & 0.42 \\
\hline$(0.980 ; 0.950) \&($ & 0.95 & 0.93 & 0.91 & 0.45 & 0.70 & 0.69 \\
\hline \multirow[t]{2}{*}{$(0.950 ; 0.950) \&(0.980 ; 0.950)$} & 72 & 0.81 & 0.79 & 1.00 & 1.00 & 1.00 \\
\hline & \multicolumn{6}{|c|}{$\mathrm{T}=3000$ and $\tau=0.8$} \\
\hline$(0.996 ; 0.990) \&(0$ & 0.19 & 0.17 & 0.15 & 0.01 & 0.01 & 0.02 \\
\hline$(0.990 ; 0.990) \&(0$ & 0.03 & 0.06 & 0.05 & 0.31 & 0.27 & 0.26 \\
\hline$(0.980 ; 0.950) \&(0$ & 0.68 & 0.60 & 0.56 & 0.16 & 0.31 & 0.30 \\
\hline \multirow[t]{2}{*}{$(0.950 ; 0.950) \&(0.980 ; 0.950)$} & 42 & 0.49 & 0.47 & 0.78 & 0.69 & 0.67 \\
\hline & \multicolumn{6}{|c|}{$\mathrm{T}=6000$ and $\tau=0.5$} \\
\hline$(0.996 ; 0.990) \&(0.990 ; 0.990)$ & 0.61 & 0.57 & 0.54 & 0.01 & 0.06 & 0.09 \\
\hline$(0.990 ; 0.990) \&(0$ & 0.08 & 0.26 & 0.28 & 0.83 & 0.74 & 0.71 \\
\hline$(0.980 ; 0.950) \&(0$ & .00 & 1.00 & 0.99 & 0.96 & 0.98 & 0.98 \\
\hline \multirow[t]{2}{*}{$(0.950 ; 0.950) \&(0.980 ; 0.950)$} & 0.99 & 0.98 & 0.98 & 1.00 & 1.00 & 1.00 \\
\hline & \multicolumn{6}{|c|}{$\mathrm{T}=6000$ and $\tau=0.8$} \\
\hline$(0.996 ; 0.990) \&(c$ & 0.34 & 0.29 & 0.27 & 0.04 & 0.05 & 0.05 \\
\hline$(0.990 ; 0.990) \&(c$ & 0.09 & 0.15 & 0.17 & 0.56 & 0.43 & 0.41 \\
\hline$(0.980 ; 0.950) \&($ & .92 & 0.88 & 0.83 & 0.67 & 0.73 & 0.70 \\
\hline \multirow[t]{2}{*}{$(0.950 ; 0.950) \&(0.980 ; 0.950)$} & 83 & 0.82 & 0.79 & 1.00 & 1.00 & 1.00 \\
\hline & \multicolumn{6}{|c|}{$\mathrm{T}=15000$ and $\tau=0.5$} \\
\hline$(0.996 ; 0.990) \&(0$. & 0.93 & 0.92 & 0.90 & 0.42 & 0.69 & 0.68 \\
\hline$(0.990 ; 0.990) \&(0.996 ; 0.990)$ & 0.71 & 0.81 & 0.80 & 0.97 & 0.94 & 0.93 \\
\hline$(0.980 ; 0.950) \&(0.950 ; 0$ & 1.00 & 1.00 & 1.00 & 1.00 & 1.00 & 1.00 \\
\hline \multirow[t]{2}{*}{$(0.950 ; 0.950) \&(0.980 ; 0.950)$} & 1.00 & 1.00 & 1.00 & 1.00 & 1.00 & 1.00 \\
\hline & \multicolumn{6}{|c|}{$\mathrm{T}=15000$ and $\tau=0.8$} \\
\hline$(0.996 ; 0.990) \&(0$. & 0.66 & 0.59 & 0.65 & 0.16 & 0.29 & 0.29 \\
\hline$(0.990 ; 0.990) \&(0.996 ; 0.990)$ & 0.39 & 0.47 & 0.46 & 0.77 & 0.68 & 0.64 \\
\hline$(0.980 ; 0.950) \&(0.950 ; 0.950)$ & 1.00 & 1.00 & 1.00 & 1.00 & 0.99 & 0.99 \\
\hline$(0.950 ; 0.950) \&(0.980 ; 0.950)$ & 1.00 & 1.00 & 1.00 & 1.00 & 1.00 & 1.00 \\
\hline
\end{tabular}


As expected, the tests display more statistical power when the structural change occur in the middle of the sample, comparatively to a more extreme position (say $\tau=0.8$ ). An interesting outcome is obtained when comparing the results for $\widehat{\sigma}^{2}$ against $\tilde{\sigma}^{2}$. It seems that the tests using $\widehat{\sigma}^{2}$ have strictly better power when the structural change is associated with a decrease in the duration of the cycles, and conversely the tests using $\widehat{\sigma}^{2}$ show more power when there is an increase in the duration of the cycles. This justifies the use of both $\widehat{\sigma}^{2}$ and $\tilde{\sigma}^{2}$ when applying the structural change tests, since for finite samples, one is suitable for the detection of increases and the other of decreases in duration of bull markets.

Comparing the three tests' statistical power one verifies that $\mathcal{D}_{1}$ introduced by Nicolau (2016) has generally more statistical power than the alternative procedures $\mathcal{D}_{2}$ and $\mathcal{D}_{3}$. The power may be low when the sample sizes are small and the duration of bull markets is high but displays suitable behavior otherwise, illustrating the consistency of the tests. In conclusion, for detecting decreases in duration in bull cycles, since the simulation results suggest that $\mathcal{D}_{1}$ with $\widehat{\sigma}^{2}$ has empirical size close to the nominal significance level considered and higher power than $\mathcal{D}_{2}$ and $\mathcal{D}_{3}$, if there is statistical evidence for rejection of $H_{0}$ by $\mathcal{D}_{1}$ a structural change is considered to have occurred.

On the other hand, simulations also show that $\mathcal{D}_{1}$ with $\tilde{\sigma}^{2}$ is serious over-sized, and therefore, in this context, the use of $\mathcal{D}_{2}$ and $\mathcal{D}_{3}$ is recommended, since the latter have considerably better empirical size behavior than $\mathcal{D}_{1}$. Thus, under this framework, a rejection of $H_{0}$ by $\mathcal{D}_{2}$ and $\mathcal{D}_{3}$ is taken as evidence that a structural break has occurred.

\section{Empirical Analysis}

\subsection{Data}

The database used in our analysis comprises adjusted market capitalization stock indexes for 18 developed and emerging markets, constructed by Morgan Stanley Capital International (MSCI) and downloaded from DataStream. For details on the construction of the adjusted market capitalization stock indexes see MSCI (2017, Appendix I). The sample sizes of the daily price indexes considered vary between 6212 and 6734 observations, due to restrictions on their availability in DataStream, with the longest samples starting 25 May 1992 and the shortest 31 May 1994. The last observed period included is 21 March 2018 for all series (see Table A.1 in the Appendix for details).

The markets considered in our analysis are the US, the UK, Canada, Belgium, Denmark, Germany, Finland, France, Ireland, Italy, the Netherlands, Norway, Spain, Sweden, Switzerland, Australia, South Africa and South Korea. ${ }^{1}$

\footnotetext{
${ }^{1}$ Japan is not included in this analysis because our focus was on countries which observed a break
} 
The classification of markets as emerging or developed followed three essential criteria: economic development, market accessibility and size/liquidity. The adjusted market capitalization stock indexes are derived from the equity universe, precisely as the investable market index. This index is then divided by the size of the companies with respect to their full market capitalization, resulting in large, mid and small cap indexes. Subsequently, for each market under analysis, the structural change tests previously described are applied to the corresponding bull market durations identified from the large, medium and small cap indexes constructed by $\mathrm{MSCI}^{2}$.

The construction of these indexes is fully described by MSCI (2017, Section 2). In particular, MSCI sorts all companies in the market investable equity universe in descending order of full market capitalization and calculates the cumulative free float-adjusted capitalization coverage for each company. Then it considers companies by market capitalization coverage of each relevant size-segment: $70 \%, 85 \%$ and $99 \%$ for the large cap, standard and investable market indexes. The mid and small caps are obtained by subtracting the large index from the standard index, and the standard index from the investable market index, respectively.

\subsection{Structural break test results}

The estimated breakpoint dates given by the structural change tests $\mathcal{D}_{1}, \mathcal{D}_{2}$, and $\mathcal{D}_{3}$, introduced in (2.5), (2.6) and (2.7), are consistent. This property is supported by the results in Bai (2000) since bull and bear markets are typically governed by a stationary first order Markov chain process, which has a first order vector autoregressive representation holding the same asymptotic properties.

To obtain robust results in our empirical analysis against possible size distortions, we identify a structural change if the null $H_{0}: \theta_{\lfloor r T\rfloor}=\theta, \forall r \in\left[r_{0}, r_{1}\right]$ is rejected by the $\mathcal{D}_{1}$, $\mathcal{D}_{2}$ or $\mathcal{D}_{3}$ tests in $(2.5)-(2.7)$ at a $5 \%$ significance level. For illustration purposes, in Figure 1 we present the application of the tests to the UK market.

due to a reduction in the duration of the bull cycle and interestingly for Japan no duration reduction was observed in the period under analysis.

${ }^{2}$ See www.msci.com/eqb/methodology/meth_docs/MSCI_June2017_GIMIMethodology.pdf. 


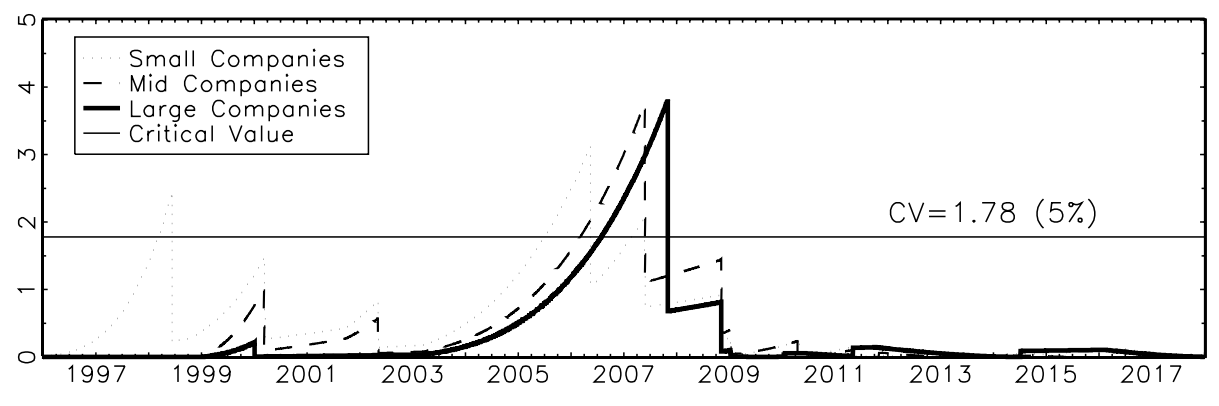

B: Duration(years)-Small Compon.

C: Duration(years)-Mid Compan.

D: Duration(years)-Large Compan.
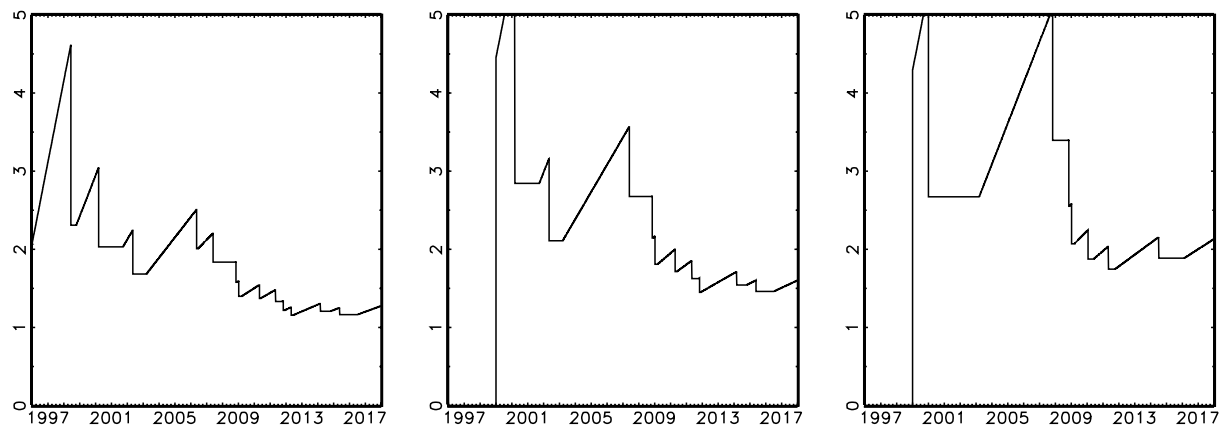

Figure 1: Illustration of application of the $\mathcal{D}_{1}$ test to the UK bull market

Panels B, C and D in Figure 1 depict the bull market durations (in years) in small, mid and large company markets. Panel A presents the results of the $\mathcal{D}_{1}$ statistics for the three markets. It is interesting to observe that: a) structural changes in the duration of the bull markets due to a decrease of duration occur before the crisis of 2008; and b) the sequence of breaks typically starts in small companies, followed by breaks in mid and large companies. The UK market illustrates what we have generally observed for other markets as well. As will be discussed in the following sections, structural changes due to a decrease in the duration of bull cycles tend to precede recession periods; and these decreases typically occur first for indexes associated with smaller companies. Table 3 and Figure 2 summarize the results obtained. Table 3 shows in detail the estimated dates for the structural changes and respective patterns. In particular, it can be observed that breaks usually happen first for smaller companies, being followed by breaks in larger companies. 


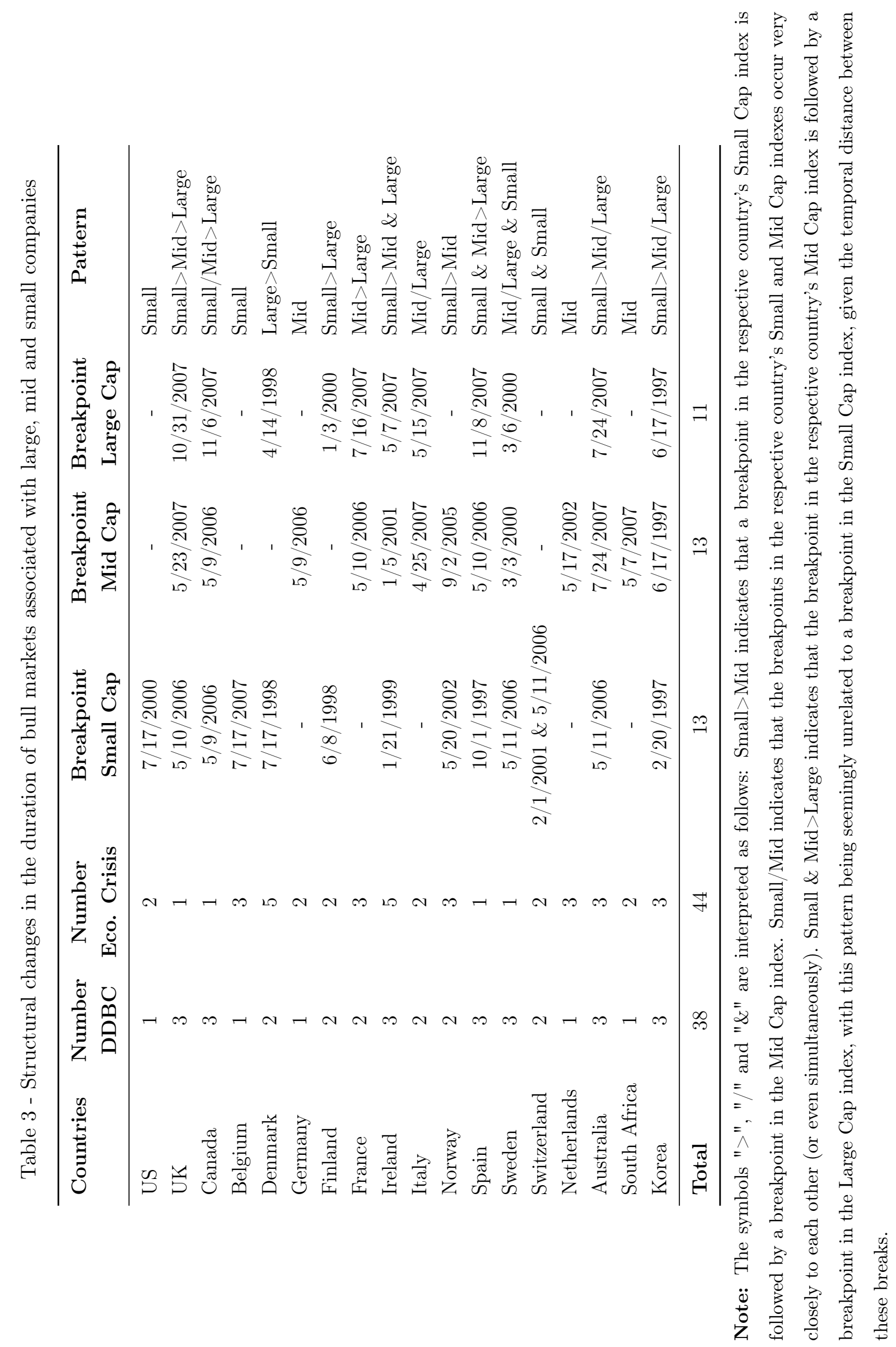



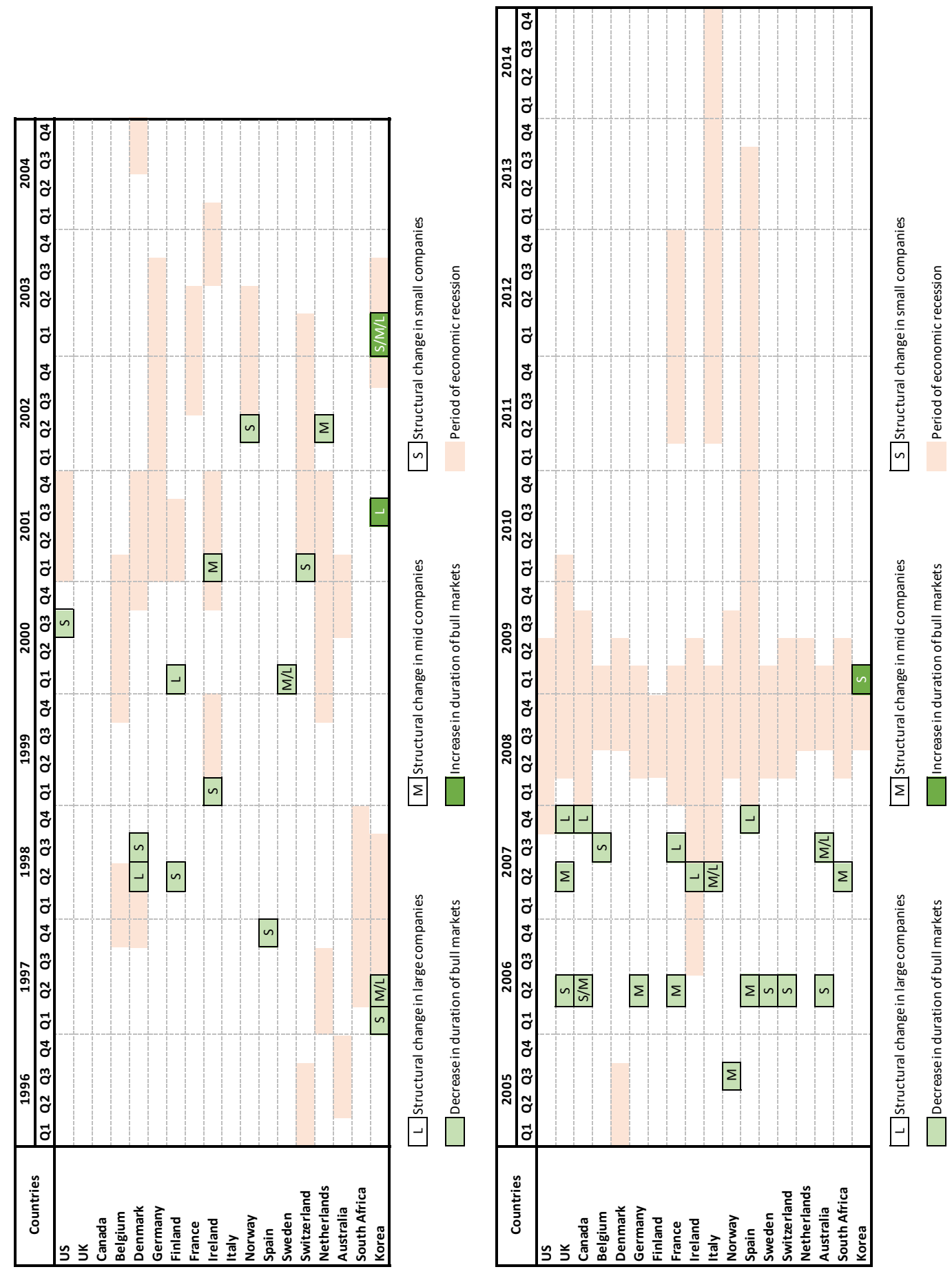

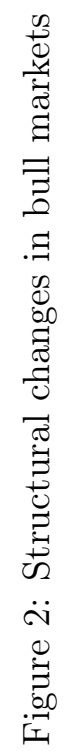


The application of the $\mathcal{D}_{1}, \mathcal{D}_{2}$ and $\mathcal{D}_{3}$ tests in (2.5), (2.6) and (2.7), respectively, reveal evidence of several structural changes in bull markets' duration between 1996 and $2014^{3}$. Upon closer inspection (see Figure 2), it becomes clear that the breaks follow some interesting patterns. Specifically, it is noticeable that decreases in the duration of bull markets (henceforth DDBC) seem to occur right before periods of economic recession. To understand this relation, consider the financial paradigm found right before the crisis of 2008, a period marked by increasing benchmark interest rates ${ }^{4}$, growing real estate bubbles and the subprime mortgage crises that significantly contributed to the decline of confidence in financial markets, backing up the popular conception that "bull markets do not die of old age, they die of fright". Additionally, Jansen and Nahuis (2003) and Fisher and Statman (2003), among others, have documented significant relations between consumer confidence and the stock market. Moreover, Chen (2011) shows that the lack of consumer confidence is associated with a higher probability of regime switching from a bull to a bear state in financial markets.

To explain the pattern observed between smaller and larger companies, notice that Kim and Burnie (2002) show that smaller companies are more vulnerable to adverse changes in economic conditions given their lower productivity and higher financial leverage. Additionally, Ehrmann (2010) points out that a monetary policy tightening, which leads to restricted access to credit by companies, is more likely to affect the smaller ones given the higher amount of collateral they have to pledge and their difficulties to access other forms of external finance, compared to larger companies.

Noticing that a monetary policy tightening actually occurred during the years anticipating the crisis of 2008, with a progressive worldwide increase in interest rates during the period before the crisis, it seems that the structural changes detected are therefore a combination of the vulnerability of smaller companies and the conditions verified over the pre-crisis period.

\subsection{DDBC and economic recessions}

The next goal is to formally analyze whether DDBC precede periods of economic recession. In what follows we use the Economic Cycle Research Institute's (ECRI) extensive chronology of business cycle peaks and troughs for our analysis; see www.businesscycle.com/ ecri-business-cycles/international-business-cycle-dates-chronologies and Fushing et al. (2010).

\footnotetext{
${ }^{3}$ No structural changes in the duration of bull markets nor economic crises were detected after 2014 for the markets included in the sample.

${ }^{4}$ See tradingeconomics.com/country-list/interest-rate for a detailed record of benchmark interest rates in the world economies.
} 
To this end we define the indicator variable,

$$
I_{i}(m):=\max \left\{I_{i}^{\text {small }}, I_{i}^{\text {medium }}, I_{i}^{\text {large }}\right\}
$$

where

$$
I_{i}^{\kappa}:=\left\{\begin{array}{ccc}
1 & \text { if } & A_{i \kappa}(m) \\
0 & \text { otherwise }
\end{array}, \text { for } \kappa=\text { small, medium or large and } i=1, \ldots, 18\right.
$$

and $A_{i \kappa}(m)$ is the event where, for the $i^{t h}$ market, a DDBC in companies of size $\kappa$ occurs $m$ months or less before a peak in the business cycle.

Under the null hypothesis that DDBC do not anticipate business cycle recessions, $\left\{I_{i}(m)\right\}$ is a sequence of i.i.d. random variables with Bernoulli distribution of parameter $p:=P\left[I_{i}(m)=1\right]$, which corresponds to the probability of at least one DDBC occurring in a given market $m$ months or less prior to an economic crisis, with both events independent of each other. The statistic that allows us to test if the structural changes detected anticipate periods of economic recession is given by,

$$
T(m):=\sum_{i=1}^{n} I_{i}(m) \sim \operatorname{Binomial}(n, p)
$$

where $n$ is the number of markets in the sample. Hence, $T(m)$ is the sum of markets which have at least one DDBC $m$ months or less before a crisis. Clearly, the greater $T(m)$, the greater the likelihood that DDBC can anticipate economic recessions. To calculate $p$ under the null hypothesis we consider the following estimator:

$$
\widehat{p}:=\sum_{x=1}^{k} \sum_{y=1}^{\infty} P[X=x \cap Y=y]\left[1-\left(\frac{T-\frac{250}{12} y m}{T}\right)^{x}\right]
$$

where $X$ is a random variable relative to the total number of DDBC associated with the small, mid and large cap indexes of a given market and $Y$ is a random variable relative to the number of economic crises experienced in the sample period. Notice that $\left[1-\left(\frac{T-\frac{250}{12} y m}{T}\right)^{x}\right]$ represents the probability that at least one of the $x$ DDBC found in the stock indexes of a given market anticipates by $m$ or less months one of its $y$ economic crises.

The probability $P[X=x \cap Y=y]$ is estimated using the markets included in the sample as,

$$
\widehat{P}[X=x \cap Y=y]=\frac{\mathrm{N}^{o} \text { of Markets verifying } \mathrm{x} \text { DDBC and } \mathrm{y} \text { crisis }}{n}
$$

where $n$ is the total number of markets in the sample verifying statistical evidence of DDBC and economic crisis.

To contrast the structural change test results and the economic crises' dates one needs information on both. The former were computed directly through the application of the 
test statistics discussed in Section 2, while the latter are obtained by considering the dates provided by ECRI when available and from Fushing et al. (2010) otherwise.

Table 4 presents the results concerning the application of the test procedure in (4.15) considering two values for $p$, one estimated as in (4.16) and the other an overestimate of this probability, $p=0.5$, more favorable to the null hypothesis of no connection between DDBC and economic crises. Considering all the scenarios specified there is strong statistical evidence that DDBC effectively anticipate periods of economic recession.

Table 4 - Binomial test results on dependence between DDBC and economic crises

\begin{tabular}{ccccc}
\hline $\mathbf{m}$ & $\mathbf{p}$ & $\mathbf{n}$ & $\mathbf{T}(\mathbf{m})$ & $\mathbf{p}$-value \\
\hline 12 & 0.1920 & 18 & 13 & 0.0000 \\
24 & 0.3473 & 18 & 16 & 0.0000 \\
12 & 0.5000 & 18 & 13 & 0.0481 \\
24 & 0.5000 & 18 & 16 & 0.0007 \\
\hline
\end{tabular}

Note: $m$ corresponds to the number of months prior to an economic crisis, $p$ is the probability, and $n$ corresponds to the total number of markets.

The estimated probabilities associated to the event in which DDBC occur $m$ months or less before an economic crisis, with both events independent are 0.19 and 0.35 for $m=12$ and $m=24$, respectively. One concludes that for the 18 markets considered which show statistical evidence of at least one DDBC and one economic crisis, 13 have at least one DDBC preceding an economic recession over the previous 12 months. This number increases to 16 if the number of months considered is 24 .

These results point to strong statistical evidence that DDBC indeed anticipate economic crisis in those countries. It seems that most markets considered have at least one DDBC preceding an economic crisis. The p-values obtained are significantly small even when using an overestimate of the probability, $p=0.5$, with the rejection of $H_{0}$ (that $D D B C$ do not precede economic crisis in the business cycle), observed for all the scenarios considered at the $1 \%$ significance level, except for $m=12$ and $p=0.5$, where the rejection is at the $5 \%$ significance level. 
Table 5 - Binomial test results on the dependence between DDBC in small, mid and large markets and economic crises

\begin{tabular}{ccccc}
\hline $\mathbf{m}$ & $\mathbf{p}$ & $\mathbf{n}$ & $T_{\text {small }}(m)$ & P-Value \\
\hline 12 & 0.1033 & 13 & 6 & 0.0011 \\
24 & 0.2056 & 13 & 9 & 0.0002 \\
12 & 0.5000 & 13 & 6 & 0.7095 \\
24 & 0.5000 & 13 & 9 & 0.1334 \\
\hline & \multicolumn{5}{c}{} \\
\hline $\mathbf{m}$ & $\mathbf{p}$ & $\mathbf{n}$ & $T_{\text {mid }}(m)$ & P-Value \\
\hline 12 & 0.1082 & 13 & 5 & 0.0009 \\
24 & 0.2164 & 13 & 9 & 0.0003 \\
12 & 0.5000 & 13 & 5 & 0.8666 \\
24 & 0.5000 & 13 & 9 & 0.1334 \\
\hline & & & & \\
\hline $\mathbf{m}$ & $\mathbf{p}$ & $\mathbf{n}$ & $T_{\text {large }}(m)$ & P-Value \\
\hline 12 & 0.0974 & 11 & 8 & 0.0000 \\
24 & 0.1948 & 11 & 9 & 0.0000 \\
12 & 0.5000 & 11 & 8 & 0.1133 \\
24 & 0.5000 & 11 & 9 & 0.0327 \\
\hline
\end{tabular}

Note: Regarding the markets considered in the analysis of the small, mid and large cap indexes see Table 3.

In order to analyse the contribution of each size index to the result previously presented, we conduct the same binomial test segregating between company size; see Table 5. With respect to the estimated probabilities associated to the event in which DDBC occur $m$ months or less before an economic crisis in small, mid and large companies, with both events independent, these are $0.10,0.11$ and 0.10 , respectively, for $m=12$, and $0.21,0.22$ and 0.19 , respectively, for $m=24$. The number of markets verifying DDBC associated with small and mid companies is the same for both sizes (13 markets); see Table 3. Hence, for the 13 markets corresponding to the small and mid companies the statistical evidence of at least one DDBC preceding an economic recession over the previous 12 months is observed for 6 and 5 markets, respectively. This number increases to 9 for both company types if the number of months considered is increased to 24 . For large companies the number of markets with evidence of DDBC is smaller, only 11, but the number of companies that show statistical evidence of having at least one DDBC preceding an economic recession over the previous 12 and 24 months ( 8 and 9 , respectively), is proportionally larger than for the small and medium companies. This happened because DDBC for large companies tend to occur after mid and small DDBC and closer to the 
date of economic crises. Interestingly, when an overestimate of the probability is considered, $p=0.5$, the null hypothesis is only rejected for large companies when at least one DDBC preceding an economic recession over the previous 24 months is considered.

\section{Conclusions}

The application of structural change tests to bull markets duration in a database comprising large, mid and small cap indexes constructed by MSCI led to the detection of several breakpoints, with our finding being the detection of a relationship between decreases in the duration of bull cycles (DDBC) and economic crises. For 13 of the 18 markets, DDBC precede at least one economic recession within 12 months. This figure increases to 16 if the larger period of 24 months is considered. Statistically, there is significant evidence that these structural changes do not occur independently from economic recessions, in fact DDBC effectively seem to precede such macroeconomic events. As we have pointed out earlier, the end of the bull cycle does not necessarily signalize a period of economic recession. However, we have found that when the end of the bull cycle is accompanied by a structural decrease in the duration of the bull cycle, a period of recession in the near future is highly probable.

Hence, monitoring financial markets with respect to bull market durations may contribute to the identification and prevention of periods of economic recession. Markets should closely monitor decreases in bull markets duration and research on this subject ought to evolve to provide methodologies to predict and better understand these structural changes.

\section{References}

Andrews, D.W.K. (1993). Tests for Parameter Instability and Structural Change with Unknown Change Point. Econometrica 61(4), 821-56.

Andrews, D. W. K. and W. Ploberger (1994). Tests for Parameter Stability and Structural Change with Unknown Change Point. Econometrica 62, 1383 - 1414.

Ang A., J. Chen, and Y. Xing (2006). Downside Risk. Review of Financial Studies, 19, 1191-1239.

Avouyi - Dovi, S. and J. Matheron (2005). Interactions between Business Cycles, Financial Cycles and Monetary Policy: Stylised Facts. BIS Papers 22, 273 - 298.

Bai, J. (2000). Vector Autoregressive Models with Structural Changes in Regression Coefficients and in Variance-Covariance Matrices. Annals of Economics and Finance $1(2), 303-339$. 
Basawa, I.V. and P. Rao (1980). Statistical Inferences for Stochastic Processes: Theory and Methods. Academic Press, London.

Candelon, B., J. Piplack and S. Straetmans (2008). On measuring synchronization of bulls and bears: The case of East Asia. Journal of Banking and Finance 32, $1022-1035$

Candelon, B., and Metiu, N. (2011). Chapter 2 linkages between stock market fluctuations and business cycles in Asia. Frontiers of Economics and Globalization, 9, 23-51.

Chauvet, M. (1999). Stock market fluctuations and the business cycle. Journal of Economic and Social Measurement, 25(3-4), 235-257.

Chauvet, M. and S. Potter (2000). Coincident and leading indicators of the stock market. Journal of Empirical Finance 7, 87-111.

Chen, S. S. (2011). Lack of consumer confidence and stock returns. Journal of Empirical Finance 18(2), 225-236.

Claessens, S., Kose, M. A. and M.E. Terrones (2012). How do Business and Financial Cycles Interact?. Journal of International Economics 87 (1), 178 - 190.

Cochran, S. J., and Defina, R. H. (1995). Duration dependence in the US stock market cycle: a parametric approach. Applied Financial Economics, 5(5), 309-318.

Durland, J. M., and T. H. McCurdy (1994). Duration-Dependent Transitions in a Markov Model of U.S. GNP Growth. Journal of Business 8 Economic Statistics 12(3), 279-288.

Ehrmann, M. (2005). Firm Size and Monetary Policy Transmission-Evidence from German Business Survey Data. IFO Survey Data in Business Cycle and Monetary Policy Analysis. Heidelberg: Physica Verlag HD, 145-172.

Estrella, A. and F.S. Mishkin (1998). Predicting US Recessions: Financial Variables as Leading Indicators. The Review of Economics and Statistics 80 (1), 45 - 61.

Fabozzi F.J., and C. Francis (1977). Stability tests for alphas and betas over bull and bear market conditions. Journal of Finance 32, 1093-1099.

Fisher, K. L., and M. Statman (2003). Consumer confidence and stock returns. Journal of Portfolio Management 30(1), 115-127.

Fort, T.C., Haltiwanger, J., Jarmin, R.S., and J. Miranda (2013). How firms respond to business cycles: The role of firm age and firm size. IMF Economic Review 61(3), 520-559.

Fushing, H., Chen, S.C., Travis, J., Berge, T.J. and Ò. Jordà (2010). A Chronology of International Business Cycles through Nonparametric Decoding. Working Paper. 
Gordon S., and P. St-Amour (2000). A Preference Regime Model of Bull and Bear Markets. American Economic Review 90, 1019-1033.

Jansen, W. J., and N.J. Nahuis (2003). The stock market and consumer confidence: European evidence. Economics Letters 79(1), 89-98.

Kim, M.K. and D.A. Burnie (2002). The Firm Size Effect and the Economic Cycle. Journal of Financial Research 25 (1), 111 - 124.

Kim M.K., and J.K. Zumwalt (1979). An Analysis of Risk in Bull and Bear Markets. Journal of Financial and Quantitative Analysis 14, 1015-1025.

Kole, E. and D.J. Van Dijk (2017). How to Identify and Predict Bull and Bear Markets?. Journal of Applied Econometrics 32, 120 - 139.

Lunde, A. and A. Timmermann (2004). Duration Dependence in Stock Prices: An Analysis of Bull and Bear Markets. Journal of Business 85 Economic Statistics 22, $253-273$.

MSCI (2017). MSCI Global Investable Market Indexes Methodology.

www.msci.com/eqb/methodology/meth_docs_MSCI_June2017_GIMIMethodology.pdf.

Naes, R., J.A. Skjeltorp, and A. Odegaard (2011). Stock market liquidity and the business cycle. Journal of Finance 66, 139-176.

Nicolau, J. (2016). Structural Change Test in Duration of Bull and Bear Markets. Economics Letters 146, $64-67$.

Nyberg, H. (2013). Predicting bear and bull stock markets with dynamic binary time series models. Journal of Banking and Finance 37(9), 3351-3363.

Pagan A.R., and K.A. Sossounov (2003). A Simple Framework for Analysing Bull and Bear Markets. Journal of Applied Econometrics 18, 23-46.

Sichel, D. E. (1991). Business cycle duration dependence: A parametric approach. The Review of Economics and Statistics, 254-260.

Sperandeo, V. (1990). Principals of Professional Speculation. New York: Wiley.

Taylor, H.M. and S. Karlin (1998). An Introduction to Stochastic Modeling. Academic Press. 
Table A.1: Sample sizes, and beginning and ending dates

\begin{tabular}{|c|c|c|c|c|}
\hline Market & Index & No. obs. & First obs. & Last obs. \\
\hline \multirow{3}{*}{ US } & Large & 6,212 & $5 / 31 / 94$ & $3 / 21 / 18$ \\
\hline & Medium & 6,212 & $5 / 31 / 94$ & $3 / 21 / 18$ \\
\hline & Small & 6,734 & $5 / 25 / 92$ & $3 / 21 / 18$ \\
\hline \multirow{3}{*}{ UK } & Large & 6,212 & $5 / 31 / 94$ & $3 / 21 / 18$ \\
\hline & Medium & 6,212 & $5 / 31 / 94$ & $3 / 21 / 18$ \\
\hline & Small & 6,585 & $12 / 31 / 92$ & $3 / 21 / 18$ \\
\hline \multirow{3}{*}{ Canada } & Large & 6,212 & $5 / 31 / 94$ & $3 / 21 / 18$ \\
\hline & Medium & 6,212 & $5 / 31 / 94$ & $3 / 21 / 18$ \\
\hline & Small & 6,585 & $12 / 31 / 92$ & $3 / 21 / 18$ \\
\hline \multirow{3}{*}{ Belgium } & Large & 6,212 & $5 / 31 / 94$ & $3 / 21 / 18$ \\
\hline & Medium & 6,212 & $5 / 31 / 94$ & $3 / 21 / 18$ \\
\hline & Small & 6,585 & $12 / 31 / 92$ & $3 / 21 / 18$ \\
\hline \multirow{3}{*}{ Denmark } & Large & 6,212 & $5 / 31 / 94$ & $3 / 21 / 18$ \\
\hline & Medium & 6,212 & $5 / 31 / 94$ & $3 / 21 / 18$ \\
\hline & Small & 6,585 & $12 / 31 / 92$ & $3 / 21 / 18$ \\
\hline \multirow{3}{*}{ Germany } & Large & 6,212 & $5 / 31 / 94$ & $3 / 21 / 18$ \\
\hline & Medium & 6,212 & $5 / 31 / 94$ & $3 / 21 / 18$ \\
\hline & Small & 6,585 & $12 / 31 / 92$ & $3 / 21 / 18$ \\
\hline \multirow{3}{*}{ Finland } & Large & 6,212 & $5 / 31 / 94$ & $3 / 21 / 18$ \\
\hline & Medium & 6,212 & $5 / 31 / 94$ & $3 / 21 / 18$ \\
\hline & Small & 6,585 & $12 / 31 / 92$ & $3 / 21 / 18$ \\
\hline \multirow{3}{*}{ France } & Large & 6,212 & $5 / 31 / 94$ & $3 / 21 / 18$ \\
\hline & Medium & 6,212 & $5 / 31 / 94$ & $3 / 21 / 18$ \\
\hline & Small & 6,585 & $12 / 31 / 92$ & $3 / 21 / 18$ \\
\hline \multirow{3}{*}{ Ireland } & Large & 6,212 & $5 / 31 / 94$ & $3 / 21 / 18$ \\
\hline & Medium & 6,212 & $5 / 31 / 94$ & $3 / 21 / 18$ \\
\hline & Small & 6,493 & $5 / 3 / 93$ & $3 / 21 / 18$ \\
\hline \multirow{3}{*}{ Italy } & Large & 6,212 & $5 / 31 / 94$ & $3 / 21 / 18$ \\
\hline & Medium & 6,212 & $5 / 31 / 94$ & $3 / 21 / 18$ \\
\hline & Small & 6,585 & $12 / 31 / 92$ & $3 / 21 / 18$ \\
\hline \multirow{3}{*}{ Norway } & Large & 6,212 & $5 / 31 / 94$ & $3 / 21 / 18$ \\
\hline & Medium & 6,212 & $5 / 31 / 94$ & $3 / 21 / 18$ \\
\hline & Small & 6,585 & $12 / 31 / 92$ & $3 / 21 / 18$ \\
\hline \multirow{3}{*}{ Spain } & Large & 6,212 & $5 / 31 / 94$ & $3 / 21 / 18$ \\
\hline & Medium & 6,212 & $5 / 31 / 94$ & $3 / 21 / 18$ \\
\hline & Small & 6,585 & $12 / 31 / 92$ & $3 / 21 / 18$ \\
\hline \multirow{3}{*}{ Sweden } & Large & 6,212 & $5 / 31 / 94$ & $3 / 21 / 18$ \\
\hline & Medium & 6,212 & $5 / 31 / 94$ & $3 / 21 / 18$ \\
\hline & Small & 6,585 & $12 / 31 / 92$ & $3 / 21 / 18$ \\
\hline \multirow{3}{*}{ Switzerland } & Large & 6,212 & $5 / 31 / 94$ & $3 / 21 / 18$ \\
\hline & Medium & 6,212 & $5 / 31 / 94$ & $3 / 21 / 18$ \\
\hline & Small & 6,585 & $12 / 31 / 92$ & $3 / 21 / 18$ \\
\hline \multirow{3}{*}{ Netherlands } & Large & 6,212 & $5 / 31 / 94$ & $3 / 21 / 18$ \\
\hline & Medium & 6,212 & $5 / 31 / 94$ & $3 / 21 / 18$ \\
\hline & Small & 6,585 & $12 / 31 / 92$ & $3 / 21 / 18$ \\
\hline \multirow{3}{*}{ Australia } & Large & 6,212 & $5 / 31 / 94$ & $3 / 21 / 18$ \\
\hline & Medium & 6,212 & $5 / 31 / 94$ & $3 / 21 / 18$ \\
\hline & Small & 6,585 & $12 / 31 / 92$ & $3 / 21 / 18$ \\
\hline \multirow{3}{*}{ South Africa } & Large & 6,212 & $5 / 31 / 94$ & $3 / 21 / 18$ \\
\hline & Medium & 6,212 & $5 / 31 / 94$ & $3 / 21 / 18$ \\
\hline & Small & 6,212 & $5 / 31 / 94$ & $3 / 21 / 18$ \\
\hline \multirow{3}{*}{ Korea } & Large & 6,212 & $5 / 31 / 94$ & $3 / 21 / 18$ \\
\hline & Medium & 6,212 & $5 / 31 / 94$ & $3 / 21 / 18$ \\
\hline & Small & 6,212 & $5 / 31 / 94$ & $3 / 21 / 18$ \\
\hline
\end{tabular}

